\title{
Impact of Same Day Screening Mammogram Results on Patient Satisfaction and Overall Breast Cancer Screening Experience - A Quality Improvement Survey Analysis
}

\author{
Biren A. Shah ( $\nabla$ Bshah@dmc.org ) \\ Wayne State University \\ Anicia Mirchandani \\ Wayne State University \\ Srishti Abrol \\ Wayne State University
}

\section{Research Article}

Keywords: screening, experience, breast, cancer, results, patients

Posted Date: November 19th, 2021

DOI: https://doi.org/10.21203/rs.3.rs-1048495/v1

License: (c) (1) This work is licensed under a Creative Commons Attribution 4.0 International License. Read Full License 


\section{Abstract}

Background: An increasing number of breast cancer cases and associated mortality due to delayed diagnosis warrants improved methods to provide a timely and convenient breast cancer screening experience.

Objective: To study the impact of same appointment mammogram results on breast cancer screening experience and patient satisfaction.

Materials and methods: A 6-question survey with questions focused on breast cancer screening experience with our new service of same appointment mammogram results was distributed to 200 patients, with 185 patients returning their responses. Patients evaluated their current experience on receiving their screening results during the same appointment with their prior breast cancer screening experience. Patients who did not respond to their satisfaction score either before or after same appointment results were excluded from the patient cohort analyzing satisfaction score. Remaining questions were analyzed separately as additional satisfaction assessment tools.

Results: About $48 \%$ of the patients indicated an improvement in their screening experience with same appointment mammography results service, while $47 \%$ of the patients reported no significant difference in their experience.

Conclusion: Although not statistically significant, same appointment mammogram results were able to make a positive impact on breast cancer screening experience among $48 \%$ of the patients. Further research elucidating barriers to screening and other ways to improve patient satisfaction will be required to increase breast cancer screening compliance.

\section{Introduction:}

In the year 2020, breast cancer in women exceeded lung cancer and has become the most commonly diagnosed cancer worldwide with over 2,261,419 new cases diagnosed in 2020 alone contributing to approximately $11.7 \%$ of all newly diagnosed cancer cases (1). Overall mortality for female breast cancer worldwide was reported to be $6.9 \%$ in 2020 (1). Breast cancer is now the leading cause of cancer death among women followed by colorectal and lung cancer (1).

Moreover, even those who have access to breast cancer screening show poor compliance with mammography. Previous studies have shown that exam compliance is related to patient experience. In a study done by Glockner et al, "personal experience" was one of the highest incentives toward getting screening mammography done. In another study by Giersch et al, part of the $54 \%$ of women who did not maintain screening adherence over three years cited decreased satisfaction with their mammography experience as one of the major contributing factors. 
The benefits of breast cancer screening, however, has consistently been demonstrated. Mortality related to breast cancer has steadily decreased in women over 50 in the past 25 years. In a metanalysis by Dibden et a/ reviewing 27 published studies on breast cancer screening programs and trials worldwide, about $22 \%$ reduction in mortality was reported with invitation to screening and $33 \%$ reduction in mortality with actual screening (2).

Although there are multiple imaging modalities such as mammography, ultrasonography and MRI, mammography remains the primary modality for initial breast cancer screening and is the only modality which has been demonstrated to reduce mortality. New guidelines by American College of Radiology (ACR) and Society of Breast Imaging (SBI) recommend annual breast cancer screening for women at average risk starting at age 40 , with evaluation of all women for high breast cancer risk starting at age 30 $(3,4)$. Despite various health organizations advocating the necessity of breast cancer screening and increased awareness among the patient population, there are multiple barriers affecting the screening rates across the world. The screening rates have been linked to fear of detection of cancer, lack of motivation and anxiety in addition to socioeconomic factors $(5,6,7)$. Behavioral changes with anxiety and feelings of depression have been reported in women in the week prior to the screening appointment (8). A study by Bull et al analyzing questionnaires filled by women at different stages of breast cancer screening from screening invitation, recall and proceeding to biopsy reported that at least $10 \%$ of the screened women suffered anxiety due to fear of having breast cancer, and about $10 \%$ of women who proceeded to undergo biopsy required psychological therapy and counselling. Multiple studies have raised concerns on screening related anxiety among women due to delay in test results stressing upon the need for expeditious and reliable screening services $(10,11,12)$.

In an attempt to reduce breast cancer screening related anxiety and test result wait times, our institution decided to provide the option for screening mammogram patients to request same day results at the time of check-in. By same day results, this meant that patients would receive their screening results and that additional diagnostic imaging would be performed on the same day of service.

Those patients that requested same day mammogram, were also asked to participate in a voluntary anonymous quality improvement survey to assess the impact it has on their screening experience.

\section{Materials And Methods:}

IRB Approval:

This study was conducted in compliance with HIPAA guidelines and was deemed exempt by the Wayne State University IRB review board after a detailed review.

Patient Cohort:

A six-question anonymous survey was given to 200 patients from June 2020 - November 2020 who opted for same day screening mammogram results at the time of check-in. 185 of those 200 patients 
completed the given survey.

For same day screening results: after completion of the mammogram, the technologist would inform the breast radiologist that the patient was waiting to receive their results. After reviewing the mammogram, the radiologist would speak to the patient in a private room to convey the results. Patients were also informed that their ordering provider would receive the mammogram report and that they can expect to receive a letter in the mail that reiterates in writing the results of their screening mammogram. After relaying the results to the patient, the patients were then asked by the radiologist whether they would be willing to complete an anonymous six-question survey regarding their experience and to then drop the survey into a locked box in the patient dressing area.

The six-question survey focused on different aspects of patient satisfaction reflecting in their responses such as average wait times following the mammogram to receive screening results, patient satisfaction with and without same day appointment result, willingness to return to our facility for their next screening mammogram, willingness to recommend our facility to their family and friends, and if they would prefer to receive results over phone at a later time than in person during the same appointment. (See supplemental file of the survey questions)

\section{Results:}

Of the 200 surveys distributed, 185 patients completed the survey. These 185 patients acted as their own controls as they were asked to compare their satisfaction level with their prior appointments when they did not receive same appointment results. Some patients chose not to answer some of the questions and those patients were excluded from analysis of each question individually.

154 patients out of the 185 patients (83.2\%) indicated they received their screening results in under 30 minutes during the same appointment. 179 patients (96.7\%) wanted to return to our facility for their next screening appointment and would recommend our facility to their friends and family. $153(82.7 \%)$ responded in favor of same appointment results rather than long wait times and delayed results over the phone.

30 patients did not provide a response to either question rating their satisfaction level with and without same appointment results due to no prior screenings to assess for the difference and were thus excluded from analysis. Therefore, we evaluated responses from the remaining 155 patients. These two questions were rated on a scale of 0-10 which provided us with a variety of responses. The satisfaction score from their prior experience was subtracted from their same day appointment satisfaction score to determine the net change in satisfaction. An improvement in screening experience was defined as a positive score: 1 or greater. No change in screening experience was defined as a score of 0 . Decreased satisfaction of screening experience was defined as a negative score.

$73(47 \%)$ of the patients reported no difference in their screening experience, with a score of 0.75 (48\%) patients reported improvement in their screening experience, with a score of 1 or greater. Although not 
statistically significant ( $\mathrm{p}$-value $\leq 0.05$ ), $48 \%$ patients reported significantly improved screening experience. It should also be noted that $7(5 \%)$ out of 155 patients reported a worse experience with same appointment screening results. (Figure 1,2)

\section{Discussion:}

Our study showed a positive impact of reduced wait times in at least $48 \%$ of patients who demonstrated improvement in their screening experience with same day appointment results but this was not shown to be statistically significant. Additionally, $47 \%$ patients showed no difference in their experience. About $5 \%$ of the patients were less satisfied with the same appointment results, which may be related to multiple factors such as patient expectations, longer than expected wait times, lack of understanding of clinic workflow and feeling pressured into waiting for result during the same appointment with not understanding their choice to reject the proposal. Of the 185 patients who filled our survey, over 154 (83.2\%) patients received their results in under 30 minutes after the mammogram and majority of the remaining patients received results in under 45 minutes which is conceivable in our clinic workflow experience. However, 4 patients (2.2\%) reported waiting time longer than an hour which may be due to the patient considering wait time starting from the time to check-in, getting the screening mammogram exam, to waiting for the results.

Willingness to return to our breast imaging center next year for a screening mammogram acted as an important assessment tool indicating that our service of same appointment results was well received by our patients with $96.7 \%$ of the patients wanting to return for future screening for same appointment results. It was also remarkable that $96.7 \%$ responded in favor of recommending our same appointment results service to their family and friends. Additionally, only $31(16.7 \%)$ patients indicated their preference of being notified of their results over phone as opposed to $153(82.7 \%)$ patients showing preference to receiving results in person than over phone. Thus, in our experience patients preferred results in person over results via phone or mail, making screening a more personable and pleasant experience for our patients. In a previous study by our group, it was evident that patients preferred results either during the same appointment or within 48 hours of the screening appointment through a follow up appointment or over the telephone being the most desirable methods (19).

Breast cancer screening can be a stressful experience for women in having to wait several days or even weeks to know the results of their screening mammogram. Sometimes, a screening mammogram result may require a recall visit for additional imaging that could take days or weeks until the patient can get their diagnostic work-up $(13,14)$. The anxiety and fear, compounded with the uncertainty and prolonged wait times, makes screening mammography an unpleasant experience which effects patient compliance rates (15). Multiple factors can further delay the breast cancer screening in different settings such as physician recommendation, lack of same-day mammography availability, lack of weekend and evening appointments for working women, lower mammography capacity of the clinics, limited notification methods, with majority of patients receiving results via mail within 30 days as per Mammography Quality Standards Act (MQSA) guidelines $(16,17,18)$. 
In a controlled trial previously done which studied the impact the availability of same day screening mammography had on patients, it was reported that same-day mammography effectively increased the adherence to breast cancer screening recommendations among women aged 50 years or older and improved patient satisfaction (17). Thus, it was again demonstrated that factors reducing the diagnostic interval have often been associated with better patient satisfaction rates in all cancer screening, reducing psychological distress and anxiety among the patients.

The probability of a patient returning for rescreening after a negative mammogram is directly related to their initial screening experience with increasing number of women avoiding rescreening if they feel dissatisfied with the service provided by the staff, longer wait times, inability to schedule appointments at a convenient time and embarrassment going through the screening (20). Breast cancer is now the most commonly diagnosed cancer as per 2020 cancer statistics and early screening is essential to detect it in early stages to reduce mortality. It can be achieved with simple steps that make breast cancer screening a less cumbersome and a patient-oriented personalized experience.

While this study showed that $48 \%$ of patient had improvement in their screening experience with same day appointment results, there were still limitations to our study. First, patients were expected to rate their satisfaction of screening experience compared to prior appointments, which could have led to recall bias. This could have been mitigated by having a control group vs those who were randomized to partake in same day appointment results. Additionally, not having a randomized control group could have subject our study to selection bias as to who voluntarily participated in same day appointment results. Lastly, our study was conducted at a single center which may make so that our data is not necessarily representative of other patient populations.

\section{Conclusion:}

Breast Cancer screening is associated with significant anxiety and distress which in turn has been shown to be related to reduced compliance in breast cancer screening. Our study sought to demonstrate whether same day appointment results could increase patient satisfaction of breast cancer screening. While a large proportion of participants reported increased satisfaction, this was not found to be statistically significant but may still have clinical utility. Further multi-institutional studies and controlled trials may highlight the benefit that same day appointment results can have on increasing patient satisfaction.

\section{Declarations:}

- Ethics approval and consent to participate - All methods were carried out in accordance with relevant guidelines and regulations. Per the Wayne State University Institutional Review board, according to Common Rule at 45 CFR 46 and FDA regulations, this project does not constitute human participant research and IRB review and oversight is not required for this project. Informed consent was obtained from all subjects involved in the study. 
- Consent for publication - Not applicable.

- Availability of data and materials - Provided. All data generated or analyzed during this study are included in this published article.

- Competing interests - The authors declare no conflict of interest.

- Funding - Not applicable

- Authors' contributions:

Dr. Biren A. Shah - was the radiologist that conveyed all the same-day results to patients and asked patients whether they would be willing to complete an anonymous survey. Contributed to the study design and survey questions. Reviewed manuscript write-up and made editing changes.

Dr. Anicia Mirchandani - Helped write-up the manuscript.

Dr. Srishti Abrol - Collected surveys and compiled the data for analysis.

- Acknowledgements:

We would like to thank Dr. Sara Sigler, PhD, Evora Vincents Sande, MBA, and Envision Healthcare Research Institute for their valuable contribution in the statistical analysis for this publication.

- Authors' information (optional):

Biren A. Shah ${ }^{1,2}$ M.D., Anicia Mirchandani, M.D. ${ }^{1}$, Srishti Abrol, M.D.. ${ }^{1}$

1- Department of Diagnostic Radiology, Detroit Medical Center, Wayne State University, Detroit, Michigan, USA.

2- Envision Healthcare

The authors declare that they had full access to all of the data in this study and the authors take complete responsibility for the integrity of the data and the accuracy of the data analysis.

\section{References:}

1. Sung, H, Ferlay, J, Siegel, RL, Laversanne, M, Soerjomataram, I, Jemal, A, Bray, F. Global Cancer Statistics 2020: GLOBOCAN Estimates of Incidence and Mortality Worldwide for 36 Cancers in 185 Countries. CA Cancer J Clin. 2020. https://doi.org/10.3322/caac.21660

2. Glockner SM, Holden MG, Hilton SV, Norcross WA. Women's attitudes toward screening mammography. Am J Prev Med. 1992 Mar-Apr;8(2):69-77. PMID: 1599723. 
3. Gierisch JM, Earp JA, Brewer NT, Rimer BK. Longitudinal predictors of nonadherence to maintenance of mammography. Cancer Epidemiol Biomarkers Prev. 2010 Apr;19(4):1103-11. doi: 10.1158/10559965.EPI-09-1120. Epub 2010 Mar 30. PMID: 20354125; PMCID: PMC2852896.

4. Dibden A, Offman J, Duffy SW, Gabe R. Worldwide Review and Meta-Analysis of Cohort Studies Measuring the Effect of Mammography Screening Programmes on Incidence-Based Breast Cancer Mortality. Cancers (Basel). 2020;12(4):976. Published 2020 Apr 15. doi:10.3390/cancers 12040976

5. Swedish Organised Service Screening Evaluation Group. Reduction in breast cancer mortality from organized service screening with mammography: 1. Further confirmation with extended data. Cancer Epidemiol Biomarkers Prev. 2006 Jan;15(1):45-51. doi: 10.1158/1055-9965.EPI-05-0349. PMID: 16434585.

6. Monticciolo DL, Newell MS, Hendrick RE, et al. Breast Cancer Screening for Average-Risk Women: Recommendations From the ACR Commission on Breast Imaging. J Am Coll Radiol. 2017;14(9):1137-1143. doi:10.1016/j.jacr.2017.06.001

7. https://www.cdc.gov/cancer/breast/pdf/breast-cancer-screening-guidelines-508.pdf

8. Ciatto S, Cecchini S, Isu A, Maggi A, Cammelli S. Determinants of non-attendance to mammographic screening. Analysis of a population sample of the screening program in the District of Florence. Tumori. 1992;78(1):22-25.

9. Austin LT, Ahmad F, McNally MJ, Stewart DE. Breast and cervical cancer screening in Hispanic women: a literature review using the health belief model. Womens Health Issues. 2002;12(3):122128. doi:10.1016/s1049-3867(02)00132-9

10. Donato F, Bollani A, Spiazzi R, et al. Factors associated with non-participation of women in a breast cancer screening programme in a town in northern Italy. J Epidemiol Community Health. 1991;45(1):59-64. doi:10.1136/jech.45.1.59

11. Walker, L.G., Cordiner, C.M., Gilbert, F.J., Needham, G., Deans, H.E., Affleck, I.R., Hood, D.B., Mathieson, D., Ah-See, A.K. and Eremin, O. (1994), How distressing is attendance for routine breast screening?. Psycho-Oncology, 3: 299-304. https://doi.org/10.1002/pon.2960030406

12. Bull AR, Campbell MJ. Assessment of the psychological impact of a breast screening programme. $\mathrm{Br}$ J Radiol. 1991;64(762):510-515. doi:10.1259/0007-1285-64-762-510

13. Mathioudakis AG, Salakari M, Pylkkanen L, et al. Systematic review on women's values and preferences concerning breast cancer screening and diagnostic services. Psychooncology. 2019;28(5):939-947. doi:10.1002/pon.5041

14. Bond $\mathrm{M}$, Pavey $\mathrm{T}$, Welch $\mathrm{K}$, et al. Psychological consequences of false-positive screening mammograms in the UK. Evid Based Med. 2013;18(2):54-61. doi:10.1136/eb-2012-100608

15. Bond M, Pavey T, Welch K, et al. Systematic review of the psychological consequences of falsepositive screening mammograms. Health Technol Assess. 2013;17(13):1-vi. doi:10.3310/hta17130

16. https://medicine.utah.edu/radiology/news/2017/04/screening-with-results.php

17. Lampic C, Thurfjell E, Bergh J, Sjödén PO. Short- and long-term anxiety and depression in women recalled after breast cancer screening. Eur J Cancer. 2001;37(4):463-469. doi:10.1016/s0959- 
8049(00)00426-3

18. Donato F, Bollani A, Spiazzi R, et al. Factors associated with non-participation of women in a breast cancer screening programme in a town in northern Italy. J Epidemiol Community Health. 1991;45(1):59-64. doi:10.1136/jech.45.1.59

19. Elkin EB, Snow JG, Leoce NM, Atoria CL, Schrag D. Mammography capacity and appointment wait times: barriers to breast cancer screening. Cancer Causes Control. 2012;23(1):45-50. doi:10.1007/s10552-011-9853-1

20. Dolan NC, McDermott MM, Morrow M, Venta L, Martin GJ. Impact of same-day screening mammography availability: results of a controlled clinical trial. Arch Intern Med. 1999;159(4):393398. doi:10.1001/archinte.159.4.393

21. https://www.fda.gov/radiation-emitting-products/regulations-mqsa/mammography-qualitystandards-act-regulations

22. Shah BA, Staschen J, Pham N, Johns A. Communicating Mammography Results: By What Method and How Quickly Do Women Want Their Screening Mammogram Results?. J Am Coll Radiol. 2019;16(7):928-935. doi:10.1016/j.jacr.2018.12.006

23. Peipins LA, Shapiro JA, Bobo JK, Berkowitz Z. Impact of women's experiences during mammography on adherence to rescreening (United States). Cancer Causes Control. 2006;17(4):439-447. doi:10.1007/s10552-005-0447-7

\section{Figures}

\section{Satisfaction}

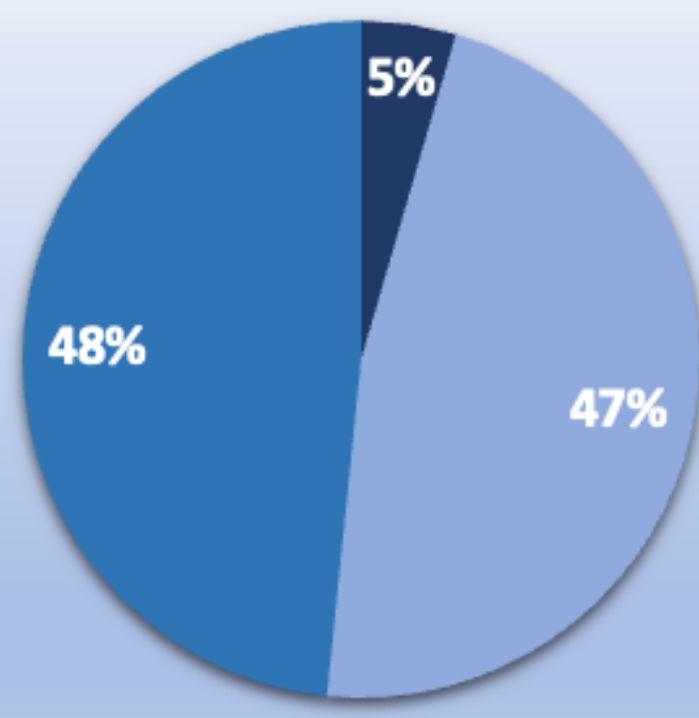

$\square$ Less Satis fied No Improvement $\quad$ More Satisfied 
Pie chart illustrating distribution of patients based on their satisfaction level after receiving same appointment screening results when compared to their old screening appointment without same day results.

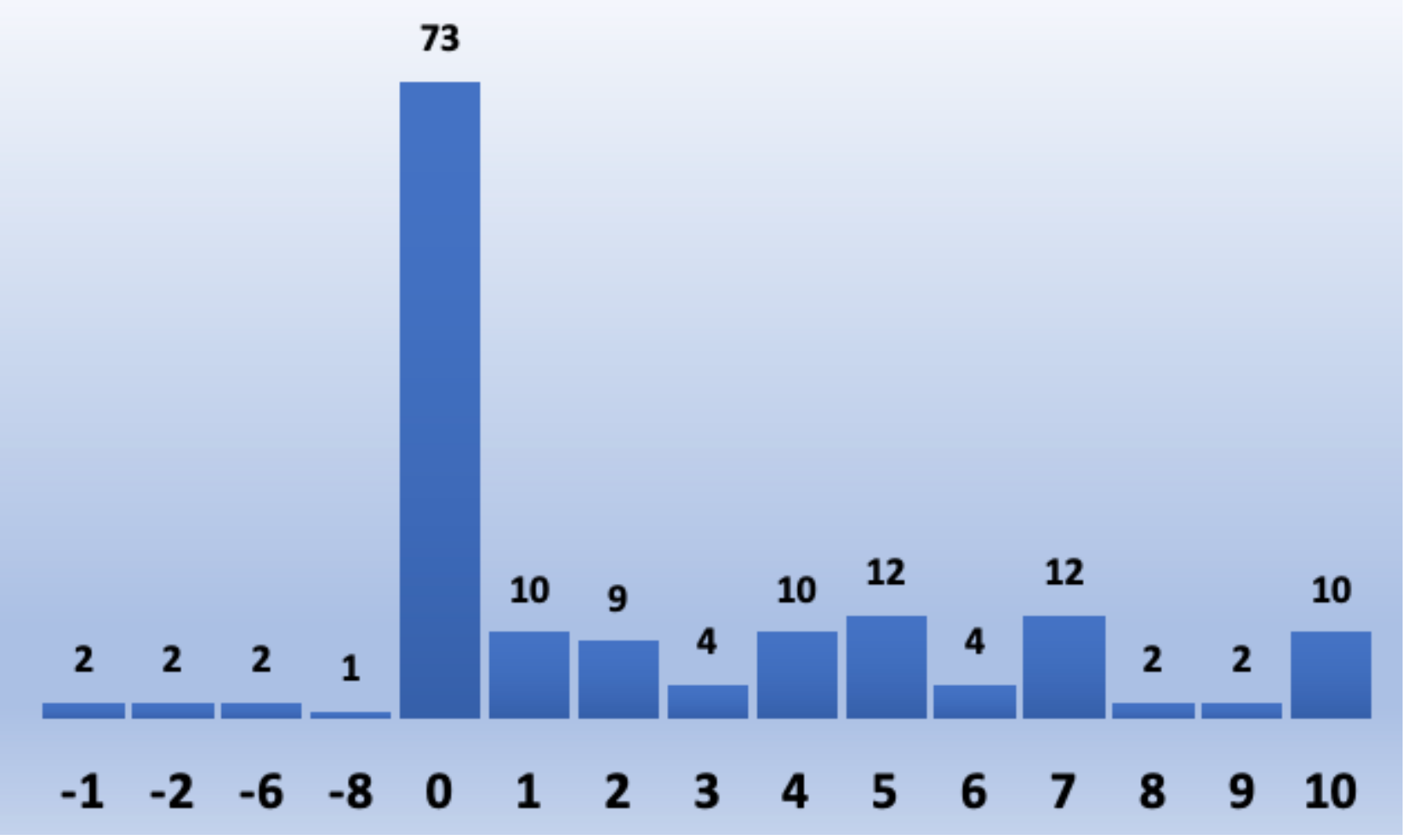

Figure 2

Histogram illustrating difference in points ranging $0 \pm 10$ estimating the change in screening experience after same appointment screening results. 0 indicates no difference in satisfaction score, below 0 indicates negative difference in satisfaction score and above 0 indicates positive difference in satisfaction score.

\section{Supplementary Files}

This is a list of supplementary files associated with this preprint. Click to download.

- Supplementalfile.docx 